\title{
Tracking from a moving platform with the Dynamic Vision Sensor
}

Cox, Joseph, Morley, Nicholas

Joseph Cox, Nicholas Morley, "Tracking from a moving platform with the Dynamic Vision Sensor," Proc. SPIE 10990, Computational Imaging IV, 1099000 (13 May 2019); doi: 10.1117/12.2518761

Event: SPIE Defense + Commercial Sensing, 2019, Baltimore, Maryland, United States 


\title{
Tracking from a moving platform with the Dynamic Vision Sensor Joseph Cox ${ }^{\mathrm{a}}$, Nicholas Morley ${ }^{\mathrm{b}}$ \\ ${ }^{a}$ College of Optical Sciences, University of Arizona, Tucson, AZ, USA \\ ${ }^{\mathrm{b}}$ Air Force Research Laboratory, Albuquerque, NM, USA
}

\begin{abstract}
The Dynamic Vision Sensor (DVS) is an imaging sensor that processes the incident irradiance image and outputs temporal log irradiance changes in the image, such as those generated by moving target(s) and/or the moving sensor platform. From a static platform, this enables the DVS to cancel out background clutter and greatly decrease the sensor bandwidth required to track temporal changes in a scene. However, the sensor bandwidth advantage is lost when imaging a scene from a moving platform due to platform motion causing optical flow in the background. Imaging from a moving platform has been utilized in many recently reported applications of this sensor. However, this approach inherently outputs background clutter generated from optical flow, and as such this approach has limited spatio-temporal resolution and is of limited utility for target tracking applications. In this work we present a new approach to moving target tracking applications with the DVS. Essentially, we propose modifying the incident image to cancel out optical flow due to platform motion, thereby removing background clutter and recovering the bandwidth performance advantage of the DVS. We propose that such improved performance can be accomplished by integrating a hardware tracking and stabilization subsystem with the DVS. Representative simulation scenarios are used to quantify the performance of the proposed approach to clutter cancellation and improved sensor bandwidth.
\end{abstract}

Keywords: Dynamic Vision Sensor, Tracking, Optical Flow, Image Stabilization, Modeling and Simulation

\section{INTRODUCTION}

This work focuses on potential approaches to improve spatio-temporal resolution in moving target tracking applications, through exploitation of the Dynamic Vision Sensor (DVS) imaging technology. These sensors generate an address-event representation $(\mathrm{AER})^{1}$ data stream from computational circuitry at the focal plane. This data stream asynchronously outputs an event at the individual pixel level when a specified log irradiance change is registered over time. The benefit of these sensors arises in the case when the background can be stabilized on the sensor (i.e. background is static) and the moving target is a small part of the overall image. In this case, the DVS only produces output at pixels corresponding to the moving target, effectively increasing the spatio-temporal resolution.

The DVS behavior can be modeled as a subtract and threshold process, which can be mathematically stated as the following decision at each pixel in a conventional video:

$$
\text { If }\left|\frac{(C S-P S)}{P S}\right|>\text { threshold, then output. Else, do not output. }
$$

where $C S$ denotes the irradiance at the current frame state, $P S$ denotes the irradiance at the previous frame state, and threshold is the percent change required to produce an output. This threshold maps to functionality similar to the DVS contrast threshold ${ }^{1}$ parameter, which can be controlled through biasing circuitry. Throughout this work, we will denote the threshold parameter as change threshold. Additional parameters can also be controlled through biasing circuitry, such as the time scale of DVS events. While this model is straightforward to understand and has been previously demonstrated ${ }^{2}$, it is not ideal, due to nonlinearities in actual DVS behavior and practicalities in acquiring conventional video. The performance of this model will be explored in Section 2.

The DVS can output using multiple schemes. The simplest scheme outputs a polarity signal at each event, providing information on whether the irradiance change is positive or negative. This was the original DVS capability ${ }^{1}$. It should be

Computational Imaging IV, edited by Abhijit Mahalanobis, Lei Tian, Jonathan C. Petruccelli, Proc. of SPIE Vol. 10990, 1099000 - (C) 2019 SPIE - CCC code: 0277-786X/19/\$18 · doi: 10.1117/12.2518761 
noted that we do not use the positive and negative polarity information, but only look at whether or not an event triggered. A more complex scheme, where the event also provides the absolute measured irradiance, is available in the Asynchronous Time-based Imaging Sensor (ATIS) technology ${ }^{3}$.

The DVS data product is in the form of an AER data stream and contains both spatial and temporal information for each event. The spatial information gives the focal plane location of the event and the temporal information gives the time of the event. Many applications asynchronously process individual events, often as soon as the DVS outputs an event. This contrasts with traditional frame-based cameras, in which an entire frame is processed as one unit. However, the individual AER events can be binned by their timestamp within a specific time interval, which converts AER events into a set of pixels within the time interval. This conversion allows the DVS data to be treated as frame-based data, which can be processed with conventional, frame-based algorithms. In this work, this conversion provides a way to compare DVS performance to frame-based camera performance.

A common DVS application is usage on a moving platform such as a car or an unmanned aerial system (UAS) to detect moving targets. In this case, the background appears to move with the platform, causing the entire image to shift on the DVS focal plane. This optical flow leads to events associated with background clutter to be added to the output, causing deleterious performance in the signal-to-clutter ratio and in spatio-temporal resolution. In this work, we seek to characterize this degradation in performance through computer DVS simulations. First, in Section 2, we discuss our DVS model, seen in Eq. (1) and similar to other methodologies ${ }^{2}$, and evaluate and discuss the model's performance. In Section 3, we introduce a new metric, Bandwidth Improvement Factor (BIF), and discuss how to use it to measure DVS performance. Next, in Section 4, we integrate the DVS model with a scene generator to understand performance in representative DVS scenarios. We then analyze the output of the scene generator in Section 5 to demonstrate the DVS performance advantage. Finally, in Section 6, we propose the integration of a motion compensation subsystem, such as a fast steering mirror, to cancel out platform motion and recapture bandwidth performance. Through this analysis, we expect to demonstrate the benefits of DVS technology, and means to achieve these benefits in practical applications.

\section{DVS MODEL}

The subtract-and-threshold model, presented in Eq. (1), allows conventional video to be converted into video representative of the DVS output data stream. In this section, we validate the model with a DVS data set, explore the accuracy of this model, and explore possible sources of residual error.

To test the model, we used a published DVS data set, which contains test scenarios recorded with the DAVIS240 DVS 4 . The DAVIS240 is a camera, with each pixel containing both a DVS output, and a conventional CMOS Active Pixel Sensor (APS) output. This means that both components share the same photodetector (with a buffer in between to stop cross talk.) Therefore, the device can simultaneously output both DVS image data and conventional CMOS camera frame data from the same focal plane.

We can apply the subtract-and-threshold process to two consecutive camera frames and compare the results to the output from the DVS. Specifically, we can bin the AER data from the DVS into a set of frames based on event timestamps. This conversion from AER data to frame data allows the DVS model to be compared to real-world data, to analyze its fidelity. In other words, if a DAVIS camera is used to measure a scene, we can compare its two outputs to determine how adequately the model represents the DVS behavior. The main caveat to this approach is that the DAVIS APS only outputs at a specified 25 frames per second (fps) ${ }^{5}$, while the DVS outputs at 12 million pixels per second. With 43,200 pixels in this camera (240x180 pixel), if there were no bandwidth improvement from the DVS, the DVS would equivalently output at $278 \mathrm{fps}$, which is much faster than the APS. Additionally, the threshold parameter was set to $30 \%$, which gave decent performance in emulating DVS behavior. It should be noted that the contrast threshold in the DVS would be biased similarly to get good performance in a scene. However, the physical DVS operates on $\log$ irradiance ${ }^{1}$, versus just irradiance as seen in the model. While we have found that the model does a good job of abstracting DVS performance, the model's subtraction step can only approximate the actual DVS's nonlinear logarithm-then-subtract step. 
The DVS database contains several different scenarios. For our analysis, we selected a scenario representative of real time aerial surveillance applications. Our specific application was with a moving, airborne platform, looking straight down with a small field of view from a constant height. This application is assumed to be in daylight. In this application, the small angle approximation is assumed to hold well, transforming translational up/down/left/right motion into tip and tilt aberrations, removing parallax effects and effectively making the scene two-dimensional.

In a scenario representative of our application ${ }^{4}$, the DAVIS was set to image a part of a large, two-dimensional printed image of a forest/rock bed. The camera moved on a rail orthogonal to the optical axis, providing a data set in which the entire image is shifted in only one dimension. Because the printed image is two-dimensional, there are no parallax effects, thus representing the airborne DVS application. In this data, the actual APS frame rate was observed to be $21.5 \mathrm{fps}$, or equivalently there was a constant $46.6 \mathrm{~ms}$ between frames. We show a sample frame from this data set in in Figure 1.

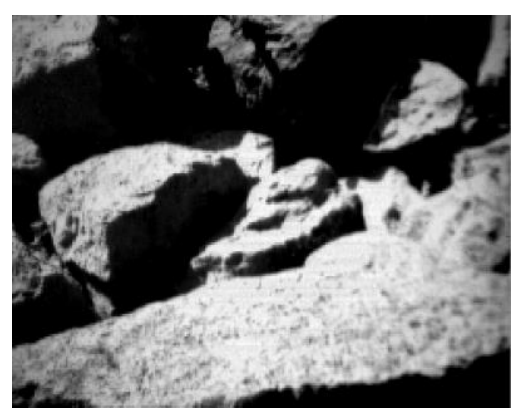

Figure 1: A sample frame from the DVS data set ${ }^{4}$. In this scenario, the camera moves on a rail to the right, causing subsequent frames and DVS outputs to appear as moving to the left.

For comparison, we applied our DVS model from Eq. (1) to the image data set, with the results shown in Figure 2. The actual DVS output is seen in Figure 3. Much of the error could be attributed to the 21.5fps frame rate in the CMOS camera. More could be attributed to a discrepancy in dynamic range and quantization error between the DVS and CMOS portions of the DAVIS240. According to the DAVIS240 specification document ${ }^{5}$, the DVS output has 120dB of dynamic range, while the CMOS camera output (Denoted as APS, or Active Pixel Sensor in the document,) has 55dB of dynamic range. This is equivalent to 3,000,000 units more of dynamic range with the DVS. The DAVIS APS images have 8-bit quantization of measured irradiance.

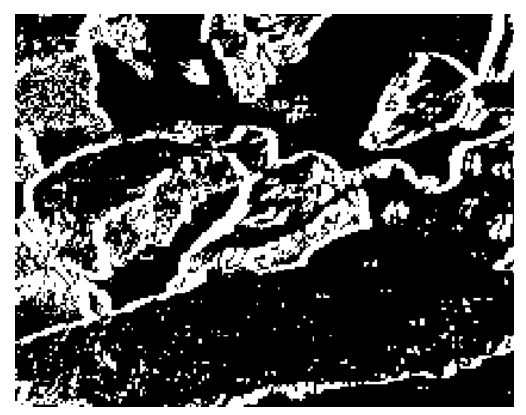

Figure 2: The output of the DVS model. Pixels with triggered events are seen in white, while pixels without events are seen in black. 


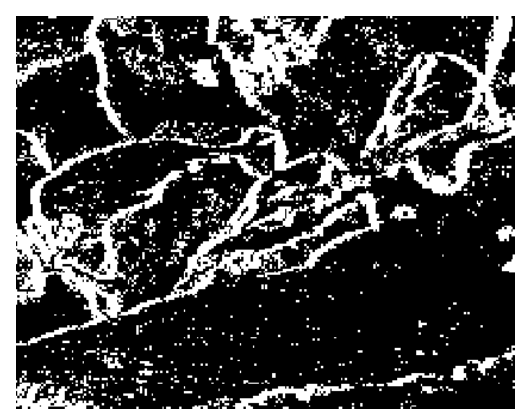

Figure 3: The actual output of the DVS, with events binned to create a frame-based representation. As in Figure 2, pixels with triggered events are seen in white, while pixels without events are seen in black.

A comparison of the images in Figures 2 and 3 is seen in Figure 4, through subtraction of the two images. This is a combination of the sets of false positive events and false negative events. Notice that there are significant differences, but that the residual appears structured. Next, through comparison of Figure 1 and Figure 4, large regions of false events are seen in darker areas of the scene. Because the represented scenario is in daylight, we can scale Figure 4 by the pixel magnitude distribution in Figure 1, to lower the weighting of dark areas in the model. If this diminishes the structured residual, we can expect this cause of error to be removed when applying the model to our daylight scenario. This hypothesis was tested, and the results are seen in Figure 5.

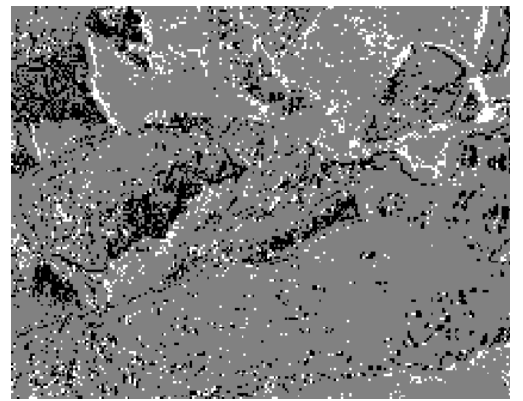

Figure 4: The residual of the difference between the model and actual DVS. Notice that there are several regions where the model works well, and several regions where it does not work well. Black pixels show that the change is detected in the model and not the actual DVS, or that the event is a false positive, while white pixels show that the change is in the actual and not the model, or that the event is a false negative. Gray pixels show that events are either true positives or true negatives.

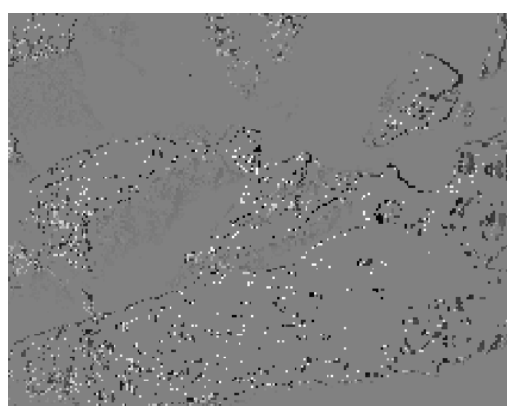

Figure 5: The residual weighted by the irradiance of the original image (Figure 1). Dark pixels correspond to false positive events and bright pixels correspond to false negative events. Gray pixels correspond to either dark areas, true positives, or true negatives. 
Looking qualitatively at the error, many potential sources of error can be described. Figure 5 suggests that dynamic range and quantization error may account for much of the residual, especially in the dark regions. Note that this residual is not likely to be detector noise, because of shot noise limited DVS behavior ${ }^{6}$. However, pictures of the experimental environment ${ }^{2}$ suggest that the scene is not particularly dark. This suggests that SNR should be sufficiently high in these areas, and that the shot noise is not the source of the error. Another source may be nonlinearity. While the model can approximate the DVS in many regions, operation on irradiance measurements in the model, as opposed to operation on the logarithm of irradiance measurements in the DVS, means that the model is only valid near a single irradiance value. Because of this, we might expect the model to more closely match the DVS in scenes with lower contrast. Additionally, Figure 5 shows sets of false events associated with contoured regions, which may be due to the $21.5 \mathrm{fps}$ APS camera. In this case, the APS output would shift by multiple pixels per frame, where the motion would appear more as a discrete shift, as opposed to the fast-responding DVS output where changes in irradiance may be smoother. Other errors include predominantly model error seen on the right side of Figure 5. As seen in Figure 1, the scene appears to get darker towards the right edge, perhaps due to vignetting effects. This gradual change in irradiance may get filtered out by the DVS differencing operation, while the model would register change from both this irradiance gradient and from actual scene motion.

We also looked quantitively at the DVS model. We ran the model on the first 50 frames of the DVS data set, at which point the data set transitions to a different scene. We then computed confusion matrix elements from the resulting set of events. These elements included true positive, true negative, false positive, and false negative event counts for each frame. The values were then used to compute an accuracy metric, or the sum of the number of true positive and negative events, divided by the total number of pixels. The accuracy, averaged across all frames, was computed as $79.9 \%$. Afterwards, the confusion matrix elements were averaged across all frames, which gave mean values of 10,612, 23,904, 4,890, and 3,794 true positive, true negative, false positive, and false negative event counts, respectively. Similar metrics could be computed with different DVS models ${ }^{2,7}$ in this scenario to compare their fidelity.

To summarize, we qualitatively analyzed error in the DVS model, and conclude that the much of the error may be explained by the DAVIS APS frame rate, DVS nonlinearity, dynamic range, and quantization error. The first can be controlled in simulation and the last two are DAVIS APS limitations which can be mitigated through usage of higher quality image sources. We then quantitatively analyzed the error, and found an average accuracy of $79.9 \%$. While this accuracy value is not optimal, qualitative discussion above suggests that much of the error may be corrected through control of the simulation, and visual comparison between Figures 2 and 3 suggests that most major features observed by the DVS were also observed in the model. Based on the above analysis, it appears that the model is adequate for our application, and for further analysis work. At this point, we moved on to higher order simulations and analysis. We believe that this decision was reasonable when applying the model to the exploration of bandwidth improvement versus decreasing platform velocity.

\section{BANDWIDTH IMPROVEMENT FACTOR}

To quantify the bandwidth improvement associated with using the DVS, we would like to know how much effective spatio-temporal resolution the DVS has, compared to a conventional camera. This metric, which we call the Bandwidth Improvement Factor (BIF), is defined as the total number of pixels output by a conventional camera in a video frame, divided by the total number of events output by a DVS, within the same time window in which a conventional camera captures a video frame.

To properly measure BIF, the DVS should have equal spatial resolution and should output the same number of events/pixels per unit time as the conventional camera. This metric is a value greater than or equal to 1 , and represents the effective improvement in spatio-temporal resolution, versus using a conventional camera. Additionally, this metric is scene dependent, and is related to the motion present in a scene. In the case of a bright, perfectly static scene, with no moving objects, BIF will be infinity, because no pixels will temporally change and shot noise will be too insignificant to cause DVS pixels to output noise. In the case of a bright, static background, with a small moving target, BIF will be very large, because only pixels corresponding to the moving target will output, and with the target being small, the DVS 
output will be very small compared to a conventional camera. In the case of a quickly moving background with large amounts of clutter, such as that seen from a quickly moving platform looking at a cluttered scene, BIF will be close to 1, because everything in the background will be moving due to optical flow, thus outputting a similar amount of data to the conventional camera.

This metric is useful because it provides a means for understanding when a DVS may be a better choice than a conventional frame-based camera for a specific application. For example, a DVS with a 1-megapixel focal plane that outputs at 1 million events per second could be compared to a conventional camera, again with a 1-megapixel focal plane, that outputs at 1 million pixels per second. In this case, the conventional camera has a frame rate of $1 \mathrm{fps}$. If the devices were compared in a scenario where the BIF is 1 , then there would be no advantage to using the DVS over a normal camera. If the BIF is 10 , then the DVS could operate at effectively 10 million pixels per second, or equivalently an effective 10fps, compared to the conventional camera. Alternately, if the spatial resolution were available, the DVS could operate a 10-megapixel focal plane while still outputting at the equivalent $1 \mathrm{fps}$ seen in the conventional camera.

It should be noted that, due to technology constraints, BIF provides an upper bound to relative DVS performance. This is because the BIF metric compares devices with equivalent spatio-temporal resolution. In practice, the DVS is a relatively new technology and has not developed to the capability of conventional cameras. For example, the DAVIS240A is specified to output at 12 million events per second, while conventional cameras can output on the order of hundreds of millions of pixels per second. If a conventional camera outputs at 120 million pixels per second, then the DAVIS240A would require a BIF of 10 to have similar performance to the conventional camera. Note that, while a conventional camera may be able to record data much faster than this, there is often a bottleneck in outputting the data to a processing system in real time.

\section{SCENE GENERATION}

The goal of this section is to provide a qualitative understanding of platform motion, and how the motion affects the DVS output. To accomplish this goal, we constructed a scene generator which is representative of our aerial surveillance application. We constructed the scene generator using public domain aerial photographs acquired from the United States Geological Survey, stitching background and target images together to simulate moving targets seen from a moving platform. These scenes include targets such as cars and trucks, as well as backgrounds such as desert, farmland, city, or grassland. Due to the realistic terrain and scenery, as well as the two-dimensional nature of the photographs, we consider the generated scenes to be representative of scenes in aerial surveillance. It should also be noted that effects such as atmospheric turbulence and platform jitter are not analyzed in this work.

After generating a video of the scene, the DVS model is applied across the entire video, using the current frame and the previous frame to compute the difference and threshold. Except where noted, the change threshold parameter has been set to $10 \%$. This contrasts with the model analysis section, where the parameter was $30 \%$. This is due to lower contrast in the scene, which requires detecting smaller changes in order to detect the target. In the following examples, the simulation is run at a 50fps frame rate. The images were captured at a 0.5 feet per pixel scale. Specifically, the background image is from the desert around Albuquerque, New Mexico, USA and the target image is from a Humvee parked at Fort Bliss, Texas, USA. The example output images in this section are specified with a rectangular full field of view of 200 by 200 pixels, or 100 by 100 feet, and with the platform moving to the left. The platform velocity is kept at low values, on the order of $<10$ feet per second, in order to represent the effectiveness of motion compensation, further discussed in Section 6 . The target is set with velocity at 20 feet per second, moving to the left. An example of the scene generation output is presented in Figure 6, which shows a sample frame of a synthetic scene with a target Humvee moving through the desert. 


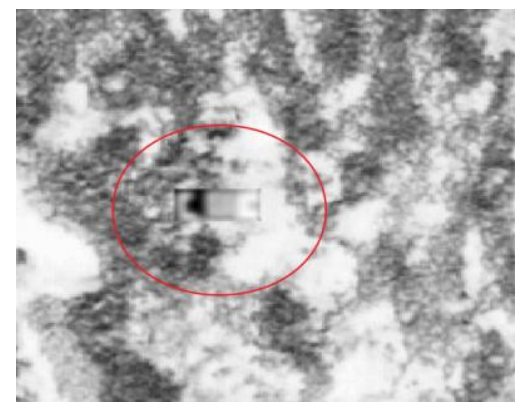

Figure 6: A sample frame from the scene generation, showing a target moving across the desert background. The target has been circled.

The DVS model is then applied to pairs of consecutive frames. We additionally simulate the ATIS capability for visualization purposes, where the location of the change, the timestamp, and the measured irradiance at that pixel are all measured, as opposed to just the location and timestamp. An example of this is shown in Figure 7, with the platform moving at 2 feet per second and the target moving at 20 feet per second. Figure 8 shows the platform moving at 4 feet per second and the target again moving at 20 feet per second.

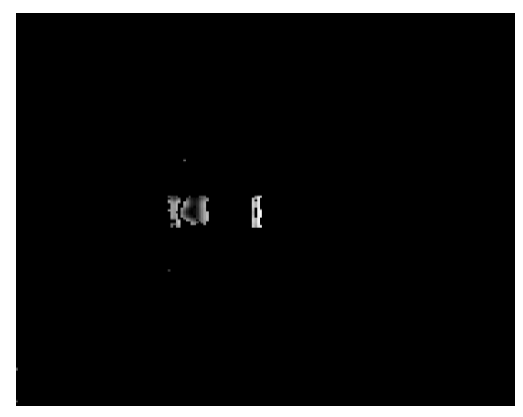

Figure 7: A frame from the simulated DVS process operating on the video in Figure 5. The platform is moving at 2 feet per second

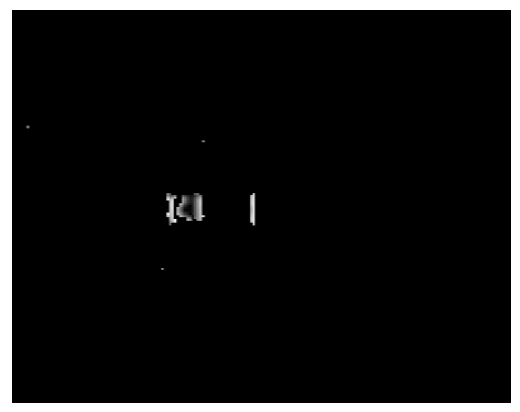

Figure 8: A frame from the simulated DVS process operating on the video in Figure 5. The platform is moving at 4 feet per second. Notice that despite the velocity increase, there is a low clutter level, similar to Figure 7.

In Figures 7 and 8, it is shown that the DVS outputs information about both the target and clutter. This clutter is generated by the optical flow from the moving platform. Both figures would have similar BIF, but Figure 8 would have slightly more. This is because while the clutter is still insignificant compared to Figure 7, the target's relative velocity compared to the platform has decreased, and thus there is less optical flow associated with the target. Also, the DVS only outputs a subset of pixels associated with the target. This is because of low spatial frequencies present in the target, which keep the change in individual pixel irradiance below the change threshold. This small subset may cause difficulties in target 
detection, due to a lack of visible features. However, it may be possible to output a larger subset of the target pixels through methods including decreasing the change threshold and increasing the time scale.

In the case of a larger platform velocity, the amount of clutter increases, due to increased optical flow. At these larger velocities, the target blends into the background, becoming more difficult to detect. As seen in Figure 9, the increased background clutter will also cause a decrease in BIF.

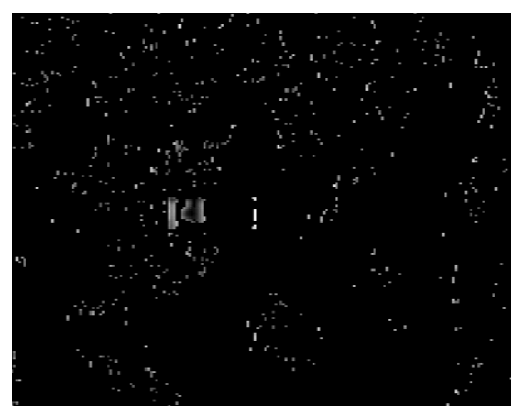

Figure 9: The target seen with a platform velocity of 8 feet per second. Notice that the clutter level has increased, which is attributed to the optical flow from platform motion.

Through comparison of Figures 7 and 9, we hypothesize that a reduction in platform velocity corresponds to a reduction in background clutter, and therefore an increase in BIF. To further illustrate our hypothesis, we generated scenes without targets. By doing so, we create images which only output pixels corresponding to background clutter. We reduced the platform velocity to show that as velocity approaches zero, DVS output clutter approaches zero. While showing the output from these scenes is somewhat of a formality, it provides context for further analysis in the following section. Figure 10 show these DVS outputs for platform velocities of 2, 4, and 8 feet per second.

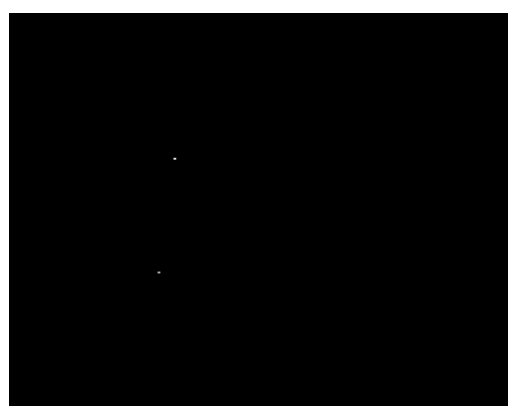

(a)

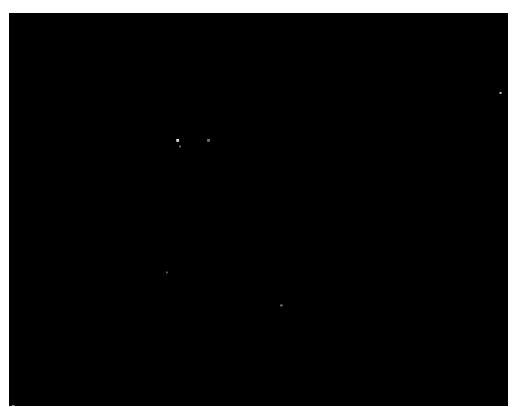

(b) 


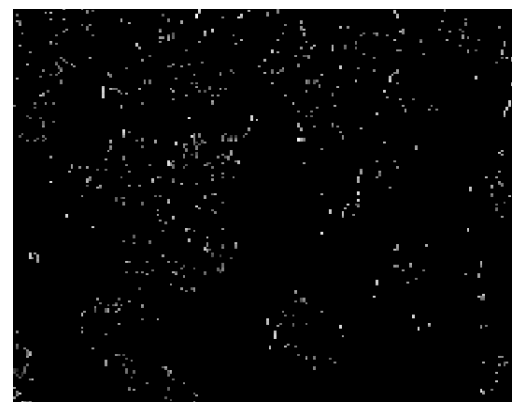

(c)

Figure 10: DVS output for a scene without targets, with (a), (b), and (c) simulated with platform velocities at 2, 4, and 8 feet per second, respectively, with the platform moving to the left.

Figure 10 demonstrates the trend of decreasing background clutter with decreasing platform velocity. The trend shows that if velocity can be sufficiently reduced when a target is present, then background clutter can be reduced to zero, leaving only moving targets as output from the DVS.

It should be noted that the effects many other parameters can be observed, but this work focuses on how platform motion affects spatio-temporal resolution and background clutter. Other parameters that we have observed include time scale (frame rate), change detection threshold, and field of view. We have observed that increasing the frame rate increases BIF, because fewer events would be triggered within the smaller time window. Increasing the field of view has been observed to cause an increase in BIF with low platform velocity. This is because the target becomes an overall smaller part of the scene, and the scene thus looks more like a scene without a target. Specifically, doubling the field of view in each direction has been observed to increase BIF by about 4 times at low platform velocity, because clutter is insignificant and the target would now be a smaller part of the output image, by a factor of 2 in each dimension, for a total factor of 4. Additionally, analysis of DVS tracking algorithm performance has been excluded, to keep the scope of this work limited.

To summarize, we generated several scenarios and applied the DVS model to the resulting video output, obtaining a qualitative understanding of the advantages of using the DVS in aerial surveillance. Specifically, we hypothesized that with lower platform velocity, the DVS has larger BIF, demonstrating significant advantage over a conventional camera in terms of spatio-temporal resolution. Then, we removed the target from the scene and illustrated more formally that the background clutter is due to platform motion. In the following section, we quantitatively analyze the platform motion to provide further evidence for our understanding of its relationship to output clutter and BIF.

\section{ANALYSIS}

The goal of this analysis is to quantitatively demonstrate the benefit of the DVS when used with a moving platform, versus a stationary platform. Additionally, this analysis demonstrates the use of BIF, and how BIF can be used to quantify DVS performance. Specifically, this analysis quantitatively shows how reduced platform velocity corresponds to a reduction in output clutter and an increase in BIF.

For this analysis, distance units are measured in feet. The images were taken at a scale of 0.5 feet per pixel. The target velocity is specified as 20 feet per second, moving to the left.

First, we examine BIF, as discussed in Section 3. This is computed as the total pixels in the image divided by the total number of DVS output pixels in a computed frame. This essentially states how much more spatio-temporal resolution the DVS has than a conventional camera. The DVS platform is specified with a rectangular, full field of view of 200 by 200 feet, or 400 pixels by 400 pixels, with its path set as entirely horizontal, moving to the left. We also varied the platform 
velocity from 1 foot per second, to 50 feet per second, in 1-foot intervals. At larger velocities, the target leaves the field of view of the platform, and thus these velocities are not included. The simulation was run at a 50fps frame rate, for 2 seconds per video, for a total of 100 frames per video. We then computed BIF for each pair of consecutive frames in a video, and averaged BIF for all pairs. This provided a single BIF value for each platform velocity. Figures 11 presents this data, consisting of BIF with and without a target, versus platform velocity.

Figure 11 provides quantitative evidence that platform velocity is inversely proportional to BIF. As platform velocity gets larger, BIF gets smaller. In the case of a present target, BIF limits at around 900 at low platform velocity. With 160,000 pixels per frame, an average of 178 pixels output at this BIF. This is because the DVS still outputs the target, which has approximately the same profile at low platform velocity. In the case of no target present, BIF continues to increase, because the target no longer limits the number of output pixels. In both cases, at low platform velocity, BIF begins to approach 1, with the slope beginning to taper off after about 10 feet per second. This is because of low spatial frequency regions in the scene. For example, in Figure 6, the bright regions consist of patches of sand and dirt, which have low spatial frequency and will have high BIF until the platform moves with high velocity. This contrasts with the dark regions, which consist of bushes, which have high spatial frequencies, and will have BIF approaching 1 as velocity increases.

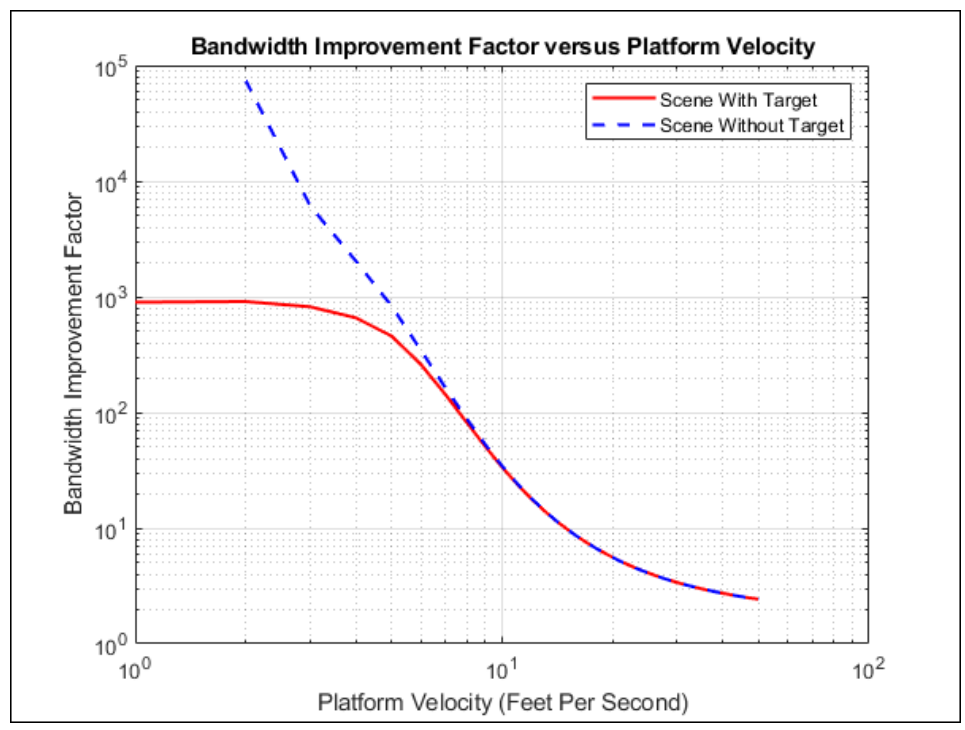

Figure 11: BIF versus platform velocity. Notice how BIF converges for higher platform velocities, but BIF is limited by targets in a scene. Also, for scenes without targets, BIF for velocity at 1 foot per second is not provided. This is because there were no events triggered at that velocity.

In the case of no target present in the scene, the entire DVS output corresponds to background clutter. In this case, the amount of clutter in the scene can be measured as the ratio of the total DVS output pixels in a frame, divided by the total number of pixels in the frame, and is equivalent to the reciprocal of BIF. This ratio gives the fraction of a frame which consists of output clutter. Thus, looking at the dotted line, background clutter is observed to be proportional to platform velocity, with the trend tapering off at higher velocities.

If, as in Section 3, a BIF of 10 is required to surpass the spatio-temporal performance of a conventional camera, we conclude that an effective platform velocity of less than 15 feet per second is required to justify usage of the DVS. In the case that velocity can be reduced to 2 feet per second, the scenario DVS would effectively have 90 times the spatiotemporal resolution of the conventional camera.

While these results are promising, this work only looks at one aspect of DVS behavior. Other aspects of the DVS data stream may prove detrimental to DVS adoption. These aspects include the sparse pixel subset associated with targets in 
the DVS output, atmospheric effects, and platform jitter, among other detriments. While other works have successfully demonstrated DVS capabilities in topics such as target recognition and tracking ${ }^{8,9,10}$, much work remains in researching DVS behavior. It should also be noted that, while the DVS model is derived and validated based on real data, simulations by themselves are not enough to prove the spatio-temporal advantage of the DVS. This additional validation effort requires hardware DVS experiments, which are due for completion in future work.

To summarize, we characterized DVS performance versus platform velocity, and quantitatively showed that BIF increases with decreasing velocity. We then showed that DVS outputs an amount of clutter proportional to the platform velocity. Given this information, we conclude that we can effectively reduce platform velocity to increase DVS performance, giving up to 90 times the spatio-temporal resolution of a conventional camera, when using assumptions from Section 3 and using system parameters shown at the beginning of the section. In the following section, we discuss how to achieve these performance improvements on a fast-moving platform such as an aircraft, through the application of motion compensation techniques.

\section{MOTION COMPENSATION}

By significantly reducing the effective DVS velocity, a large BIF can be realized. This would give the DVS a significant advantage over a conventional, frame-based camera.

If the DVS has a sufficiently small field of view and is sufficiently far from the scene, then a small angle approximation can be used on the scene. In this case, translational motion orthogonal to the optical axis can be approximated as a tip or tilt aberration. Therefore, if this aberration is corrected (preferably in the optical domain), orthogonal platform motion could be compensated for and canceled out, realizing the bandwidth improvement in the DVS.

Several methods exist to implement this motion compensation technique. In each method, the objective is to stabilize the image on the focal plane through cancellation of tip and tilt aberrations. First, motion compensation can be performed on the focal plane in image space, as in the case of MIT Lincoln Laboratory's Orthogonal Transfer capability ${ }^{11}$. With this capability, as with many photodetectors, each pixel stores an electric charge, whose magnitude is proportional to the measured irradiance from a location in a scene. This charge can be shifted to neighboring pixels in the up/down and left/right directions, which is equivalent to applying a tip or a tilt aberration in object space. Other imaging systems with similar focal plane shift capabilities include the SCAMP vision sensor ${ }^{12}$ with its interpixel communications functionality. Motion compensation can also be implemented optically, in object space, as in the case of a fast steering mirror. In this case, the fast steering mirror directly compensates for tip and tilt introduced by motion.

The motion compensation technique relies on having accurate information on platform velocity and distance from the scene, and being able to relay this information to the motion compensation system. Here, the collected information should be combined to compute the expected tip and tilt values introduced by motion, and then converted to a pixel shift or directly input into an optical compensation system.

Motion compensation can be applied using a staring technique. As the platform moves along a path, after a short period of staring at a single scene location, the motion compensation system should be reset to look at other locations. In other words, the function of the brief staring period is to provide a window to observe moving objects in part of the scene, while still having the capability of viewing other parts of the scene.

The information for motion compensation can come from several sources. For example, platforms such as aircraft may have subsystems that can provide this information through an interface to the motion compensation subsystem. Another possibility could be to use a closed-loop system with the DVS, selecting motion compensation values based on output DVS data, with the objective of maximizing BIF. In other instances, self-contained measurement devices such as GPS or IMU can provide the necessary information. However, this information may be insufficiently accurate for realizing the DVS bandwidth improvement. For example, as seen in Figure 11, motion compensation is insufficient when it can only compensate to an accuracy of 15 feet per second. In this case, the bandwidth improvement alone would not justify using 
the DVS. However, if the system could compensate to 2 feet per second, then the DVS could realize its potential, and the tracking system would benefit from the 90x bandwidth improvement, also seen in Figure 11.

\section{SUMMARY}

We investigated DVS behavior through software simulation applied to moving target tracking applications. We analyzed our DVS model, and determined that its behavior was an adequate representation of DVS behavior for our applications. We then defined a new metric, BIF, to characterize relative DVS performance in a specific application when compared to a conventional camera. We discussed how using BIF, or the reduction of bandwidth required to detect a moving target, could be used to improve an imaging system, gaining higher spatial resolution through a larger focal plane, higher temporal resolution through faster processing times, or both. Next, we constructed a scene generator to simulate moving target tracking in a representative scenario, and analyzed the effect of platform motion on the application. Finally, we concluded that, by integrating a motion compensation system such as a fast steering mirror, we could potentially reduce effective platform velocity seen by the DVS, creating conditions where the DVS has a large significant spatio-temporal benefit over a conventional camera.

\section{ACKNOWLEDGMENT}

The images from Albuquerque, NM, USA and Fort Bliss, TX, USA are courtesy of the U.S. Geological Survey.

\section{REFERENCES}

[1] P. Lichtsteiner, P., C. Posch, and T. Delbruck, "A 128x128 120dB 15 $\mu$ s latency asynchronous temporal contrast vision sensor”, IEEE J. Solid-State Circuits, 43(2):566-576, 2008.

[2] J. Kaiser, J. C. V. Tieck, C. Hubschneider, et. al, "Towards a framework for end-to-end control of a simulated vehicle with spiking neural networks", 2016 IEEE International Conference on Simulation Modeling and Programming for Autonomous Robots (SIMPAR), pp. 127-134, Dec 2016.

[3] C. Posch, D. Matolin, and R. Wohlgenannt (2011). "A QVGA 143 dB Dynamic Range Frame-Free PWM Image Sensor With Lossless Pixel-Level Video Compression and Time-Domain CDS”, IEEE J. Solid-State Circuits, 46(1):259275, 2011.

[4] E. Mueggler, H. Rebecq, G. Gallego, et. al., "The Event-Camera Dataset and Simulator: Event-based Data ...”, International Journal of Robotics Research, Vol. 36, Issue 2, pp. 142-149, Feb. 2017

[5] iniVation AG "DVS and DAVIS Specifications", www.inivation.com

[6] Lichtsteiner, P., "A temporal contrast vision sensor", Ph.D. Thesis, ETH Zurich, Zurich, Switzerland. p. 92. 2006;

[7] M. L. Katz, K. Nikolic, and T. Delbruck, "Live demonstration: Behavioural emulation of event-based vision sensors," in Circuits and Systems (ISCAS), 2012 IEEE International Symposium on, May 2012, pp. 736-740.

[8] A. Mitrokhin, C. Fermuller, C. Parameshwara, Y. Aloimonos, "Event-based moving object detection and tracking," Proc. IEEE/RSJ Int. Conf. Intell. Robot. Syst., pp. 1-9, Oct. 2018.

[9] D. Drazen, P. Lichtsteiner, P. Häfliger, et. al.,"Toward real-time particle tracking using an event-based dynamic vision sensor," Exp. Fluids, vol. 51, no. 5, pp. 1465-1469, Nov. 2011.

[10] Cohen G., Afshar S., van Schaik A., et al., "Event-based Sensing for Space Situational Awareness," in Advanced Maui Optical and Space Surveillance Technologies Conference (AMOS) (Maui, HI), pp. 1-13. 2007.

[11] J. Tonry, B. E. Burke, and P. L. Schechter, "The Orthogonal Transfer CCD," PASP 109, pp. 1154-1164, Oct. 1997

[12] S. J. Carey, D. R. W. Barr, A. Lopich, and P. Dudek, "A 100,000 fps Vision Sensor with Embedded 535 GOPS/W

256x256 SIMD Processor Array”, VLSI circuits symposium, Kyoto, Japan, pp.C182-C183, June 2013. 